\title{
Postoperative Doxycycline Did Not Improve Early Postoperative Outcomes in Hip Arthroscopy Patients: A Retrospective Cohort Study
}

\author{
Zeeshan M. Akhtar, MD ${ }^{1}$ Emily R. Hunt, PhD ${ }^{1} \quad$ Brooks N. Platt, MD ${ }^{1}$ Alex L. Turner, BS ${ }^{1}$ \\ Cale A. Jacobs, PhD ${ }^{2}$ Austin V. Stone, MD, PhD ${ }^{1}$ Stephen T. Duncan, MD ${ }^{1}$ \\ ${ }^{1}$ Department of Orthopaedics, University of Kentucky, Lexington, \\ Kentucky \\ 2 Department of Orthopaedic Surgery \& Sports Medicine, University \\ of Kentucky Medical Center, Lexington, Kentucky \\ Address for correspondence Stephen T. Duncan, MD, Department of \\ Orthopaedics, University of Kentucky, 125 E Maxwell St. Suite 201, \\ Lexington, KY 40508 (e-mail: stdunc2@uky.edu). \\ J Hip Surg 2022;6:8-14.
}

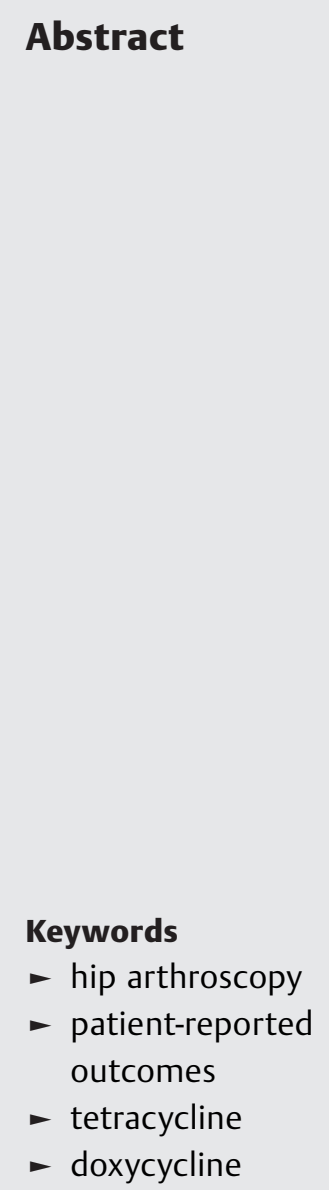

Doxycycline has been shown to reduce fibroblast activity in the treatment of multiple pathologies, and was utilized as part of the postoperative medication protocol to help prevent adhesions from developing after hip arthroscopy. The purpose of this study was to compare early postoperative pain outcomes between consecutive case series of femoroacetabular impingement syndrome (FAIS) patients treated with or without a 7day postoperative course of doxycycline following hip arthroscopy. We hypothesized that the doxycycline-treated group would have superior patient-reported outcomes at 6 weeks, and the effect would be more pronounced for patients with low preoperative mental health scores.

Our institutional review board-approved prospective outcomes registry was queried for patients who had undergone arthroscopic femoral osteochondroplasty and/or labral repair or reconstruction secondary to FAIS performed by a single, fellowshiptrained surgeon. A 7-day postoperative course of doxycycline was initiated in 2018. Sixweek postoperative Hip Disability and Osteoarthritis Outcome Score (HOOS) global $\left(\mathrm{HOOS}_{\text {global }}\right)$ and International Hip Outcome Tool (iHOT) scores were compared between the consecutive series treated with versus without doxycycline. Additional subgroup analysis was performed on patients with low preoperative Veterans RAND 12Item Health Survey (VR-12) mental component scores to determine possible additional benefit of postoperative doxycycline in this group.

A total of 134 patients ( 98 female, 36 male, age $=36.2 \pm 13.4$ years, body mass index $\left.[\mathrm{BMI}]=27.2 \pm 5 \mathrm{~kg} / \mathrm{m}^{2}\right)$ were identified between 2013 and 2020. There were no differences between the control $(n=84)$ and doxycycline group $(n=50)$ in age, sex, or BMI. Postoperative HOOS $_{\text {global }}$ scores (control $=58.5 \pm 13.1$, doxycycline $=58.7 \pm 14.7, p=0.96$ ) and iHOT scores (control $=54.4 \pm 22.2$, doxycycline $=52.3 \pm 24.8, p=0.61$ ) did not differ between groups. The subset of 83 patients with low preoperative mental scores showed received

April 24, 2021

accepted

October 6, 2021

published online

November 18, 2021 (c) 2021. Thieme. All rights reserved.

Thieme Medical Publishers, Inc.,

333 Seventh Avenue, 18th Floor,

New York, NY 10001, USA
DOI https://doi.org/

10.1055/s-0041-1739456. ISSN 2472-8446. 
no differences in $\mathrm{HOOS}_{\text {global }}$ (control $=51.6 \pm 13.3$, doxycycline $=57.3 \pm 7.4, p=0.12$ ) or iHOT scores (control $=45.5 \pm 20.3$, doxycycline $=53.6 \pm 23.1, p=0.250$ ).

Our results do not support the hypothesis that postoperative doxycycline leads to significant improvement in early postoperative pain scores or functional outcomes in patients undergoing hip arthroscopy for FAIS.

Femoroacetabular impingement syndrome (FAIS) describes a condition in which the anatomy of the hip allows abnormal contact between the femoral head and the acetabulum. FAIS may lead to pain, cartilage damage, and labral tears, resulting in hip dysfunction. ${ }^{1}$ FAIS is associated with two main anatomical deformities: the cam deformity and the pincer deformity. The cam deformity is associated with an increased $\alpha$ angle, and is described as an unnaturally aspherical shape of the femoral head. The pincer deformity is described as overcoverage of the femoral head by the acetabulum, and is associated with lateral center-edge angle (LCEA). ${ }^{1}$

While open surgical treatment has been reported to be successful in treating FAIS, ${ }^{1}$ hip arthroscopy has evolved into the primary surgical option for the treatment of FAIS. Systematic reviews comparing the arthroscopic approach to open procedures have demonstrated arthroscopic procedures to be a safe and effective method of treatment, with a reported complication rate of 0 to $5 \% .^{2}$ Specifically, persistent postoperative pain and restricted range of motion, which may be caused by postoperative adhesion formation, ${ }^{3}$ are of exceeding importance in the young, active FAIS population. ${ }^{4}$ Adhesions form as fibrin deposits secondary to the coagulation cascade and lead to fibroblast chemotaxis, which deposit the extracellular matrix (ECM). ${ }^{5}$

Resolution of postoperative pain and discomfort continues to be a limiting factor in patient-reported outcome (PRO) scores. Numerous modalities have been evaluated to combat postprocedural pain in this population, including perioperative nerve blocks and local anesthetic injections as well as preoperative anti-inflammatory medications. ${ }^{6}$ While not yet labeled for such a use, doxycycline has several potential mechanisms to reduce postoperative pain after hip arthroscopy, including inhibiting painful adhesions. Many adhesions are asymptomatic, but some can be a significant source of postoperative pain. ${ }^{3}$ The pathogenesis of arthrofibrosis is largely due to the activation and differentiation of fibroblasts. ${ }^{7}$ Doxycycline reduces fibroblast activity and the inflammatory dysregulation of matrix metalloproteinases (MMPs) that can lead to ECM contraction in a variety of pathologies. ${ }^{8-10}$ In addition to inhibiting painful adhesions, the anti-inflammatory effects of tetracyclines have been shown to suppress secondary pain pathways, resulting in reduced central nervous system inflammation. ${ }^{11}$ However, the literature is sparse in documenting the modulation of the neuroinflammatory response in the surgical setting.

The modulation of secondary pain pathways is of particular interest in the hip arthroscopy patient population that has higher prevalence of chronic opioid use, substance abuse, and concomitant psychiatric conditions such as anxiety and depression. ${ }^{12}$ Furthermore, anxiety and depression are independently predictive of poor PROs and postoperative pain scores in patient undergoing hip arthroscopy. ${ }^{13}$ The neuromodulatory and secondary pain pathway effects of doxycycline may have an added benefit in this patient population. This may be specifically measured in common PROs in questions that specifically relate to pain and in functional measures that may improve due to decreased pain.

The purpose of this retrospective case-control study was to determine if those who underwent a hip arthroscopy procedure for femoroacetabular instability (FAIS) and took a 7-day postoperative course of doxycycline had improved PRO scores compared with those who did not take doxycycline postoperatively. Our hypothesis was that postoperative doxycycline $100 \mathrm{mg}$ twice daily for 7 days would improve early PROs after hip arthroscopy. We secondarily hypothesized that this effect would be intensified in those patients with a mood disorder.

\section{Methods and Materials}

The University of Kentucky institutional review board-approved prospective hip arthroscopy outcomes registry was queried for patients who had undergone arthroscopic femoral osteochondroplasty and/or labral repair or reconstruction secondary to FAIS performed by a single, fellowship-trained surgeon between December 30, 2013, and May 26, 2020. Patients were excluded if they underwent open hip procedures, revision hip arthroscopy, or the indication for surgery was anything other than FAIS. Starting in November 2018, patients with FAIS treated with an arthroscopic procedure were given a 7-day course of doxycycline ( $100 \mathrm{mg}$ twice daily).

Preoperative Veterans RAND 12-Item Health Survey (VR-12) mental component scores (MCSs), 6-week postoperative Hip Disability and Osteoarthritis Outcome Score (HOOS) global ( HOOS $_{\text {global }}$ ), and International Hip Outcome Tool (iHOT) scores were then compared between the consecutive series treated with versus without doxycycline. Additional subgroup analysis was performed on patients with low preoperative VR-12 MCSs to determine possible additional benefit of postoperative doxycycline in this group. Consistent with previous studies, the threshold to categorize patients as having low preoperative VR-12 MCSs was a score of less than $42.9 .^{14,15}$

Demographic measures collected included age, sex, and body mass index (BMI). Radiographic measures collected included pre- and postoperative $\alpha$ angle and LCEA. Alpha angles were measured by drawing a best-fit circle around the femoral head in either the Dunn view or the frog-leg lateral 
view of the operative leg. One component of the angle was made by a line in the trajectory of the femoral neck running through the center of the best-fit circle. The second component was comprised of the line from the center of the best-fit circle to the point at which the femoral head or neck exceeds the circumference of the best-fit circle. ${ }^{16}$ The LCEA was measured in the standing anteroposterior pelvis view by drawing a best-fit circle around the femoral head. The angle was formed by the line perpendicular to the horizontal axis through the center of the best-fit circle and a line intersecting with the lateral edge of the acetabulum. ${ }^{17}$ Operative data collected included number of anchors used, Outerbridge classification of femoral, acetabular chondromalacia observed during arthroscopy, and size of labral tear visualized during arthroscopy as measured on a clock face. Further postoperative variables collected included number of reoperations.

Proportions of patients who achieved a patient-acceptable symptom state (PASS) were calculated for each group and compared in both the overall and subgroup analysis. PASS is defined by a HOOS $_{\text {global }}$ score of at least 62.5, which was calculated to represent the threshold at which the majority of patients were satisfied with their procedure. ${ }^{18}$ With the primary end point of proportion achieving the HOOS $_{\text {global }}$ threshold, a sensitivity analysis was performed to determine the minimum percentage difference detectable with the existent data. With $\alpha$ set at 0.05 and $\beta$ at 0.80 , the cohort of 132 patients who completed a postoperative $\mathrm{HOOS}_{\text {global }}$, the minimum PASS threshold proportion difference detectable was about $23.5 \%$. In addition, to isolate a postoperative measure of pain, a component of the iHOT was analyzed separately, which consisted of a 100-point visual analogue scale (VAS) for pain, labeled in the following as iHOT VAS.

Two-sample $t$-tests were used to compare the postoperative outcome means. The z-test for proportions was used to analyze the difference between the proportion of patients who achieved the PASS threshold and those who underwent reoperation. A chi-square test was used to determine differences between the doxycycline and no-doxycycline groups in terms of Tonnis and Outerbridge classifications. R software version 4.0.2 ( $\mathrm{R}$ Foundation for Statistical Computing, Vienna, Austria $)^{19}$ was used for data analysis. Statistical significance was set at $p<0.05$.

\section{Results}

\section{Demographics}

In total, 134 patients were identified who underwent hip arthroscopy for FAIS and completed either postoperative
iHOT or postoperative HOOS $_{\text {global }}$ surveys. The average age of all patients was $36.2 \pm 13.4$ years. There were 98 female patients (73.1\%), and the average BMI was $27.2 \pm 5 \mathrm{~kg} / \mathrm{m}^{2}$. The group that received doxycycline did not significantly differ from the group that did not receive doxycycline in age, sex, or BMI (-Table 1). The groups also did not differ in distribution of acetabular versus femoral osteochondroplasty, labral repair versus reconstruction, frequency of capsular closure, or use of knotless versus knotted anchors (-Table 2).

\section{Overall Outcomes}

Preoperative MCSs were significantly lower in the group that received doxycycline (38.2 vs $48.8, p<0.001$ ). However, there was no significant difference in preoperative $\alpha$ angle, LCEA, or Tonnis grade (-Table $\mathbf{3}$ ). In addition, there was no difference in average number of anchors used intraoperatively, nor was there a difference in the Outerbridge grade of acetabular or femoral chondromalacia described intraoperatively. Ultimately, the groups did not differ significantly with respect to postoperative iHOT, HOOS global, $\alpha$ angle, LCEA, iHOT VAS, the proportion above HOOS $_{\text {global }}$ PASS threshold, or reoperation rate ( - Table 4 ).

\section{Subgroup Analysis}

Among those with low preoperative MCSs ( $<42.9, N=83)$, the groups did not differ with respect to any postoperative outcome, including proportion above $\mathrm{HOOS}_{\text {global }}$ PASS threshold and reoperation rate. However, while pre- and postoperative LCEAs were not significantly different between the two groups, $\alpha$ angles were lower on average in both timeframes in the group that received doxycycline (55.3 vs 63.5 degrees, $p=0.009 ; 44.3$ vs 48.7 degrees, $p=0.042$ ). Additionally, more anchors were used on average in the doxycycline group (four vs three, $p=0.018$ ) (-Table 5 ).

\section{Discussion}

The purpose of this study was to assess the potential benefits of postoperative doxycycline use in the FAIS population treated with hip arthroscopy. The primary finding of this study was that a 7-day course of postoperative doxycycline did not improve early clinical outcome scores after hip arthroscopy, regardless of preoperative mental health status.

This study is the first to examine the effects of doxycycline use in modifying postoperative pain. Tetracyclines, while initially used for their bacteriostatic effects, have recently been studied for their antihypernociceptive and anti-inflammatory effects. ${ }^{20}$ The anti-inflammatory effects of another

Table 1 Group demographics

\begin{tabular}{|l|l|l|l|}
\hline & Doxycycline $(\boldsymbol{n}=50)$ & No doxycycline $(\boldsymbol{n}=\mathbf{8 4})$ & $\boldsymbol{p}$-Value \\
\hline Age $(\mathrm{y})$ & $35.9 \pm 11.2$ & $35.5 \pm 11.4$ & 0.840 \\
\hline Sex $(\mathrm{M} / \mathrm{F})$ & $16 / 34$ & $20 / 64$ & 0.410 \\
\hline Body mass index & $26.9 \pm 5.1$ & $27.4 \pm 4.9$ & 0.580 \\
\hline
\end{tabular}


Table 2 Procedure breakdown and analysis; Fisher's exact test for osteochondroplasty breakdown, repair versus reconstruction, knotless versus knotted anchors, and specific anchor types; z-test for proportions for percentage of capsular closure

\begin{tabular}{|c|c|c|c|}
\hline & Doxycycline $(N=50)$ & No doxycycline $(N=84)$ & $p$-Value \\
\hline \multicolumn{4}{|l|}{ Osteochondroplasty location } \\
\hline Acetabular osteochondroplasty & 0 & 13 & \multirow[t]{2}{*}{$>0.999$} \\
\hline Femoral osteochondroplasty & 48 & 81 & \\
\hline \multicolumn{4}{|l|}{ Labral treatment } \\
\hline Labral repair & 50 & 71 & \multirow[t]{2}{*}{$>0.999$} \\
\hline Labral reconstruction & 8 & 8 & \\
\hline Capsular closure (\%) & $49(98.0)$ & $82(97.6)$ & 0.889 \\
\hline \multicolumn{4}{|l|}{ Knotted versus knotless } \\
\hline Knotted anchors & 31 & 73 & \multirow[t]{2}{*}{0.538} \\
\hline Knotless anchors & 1 & 1 & \\
\hline \multicolumn{4}{|l|}{ Specific anchor types } \\
\hline Pivot NanoTack & 26 & 67 & \multirow[t]{7}{*}{0.514} \\
\hline Smith and Nephew Bioraptor & 0 & 3 & \\
\hline Smith and Nephew Healicoil and Footprint & 0 & 1 & \\
\hline Smith and Nephew Suture Fix & 0 & 2 & \\
\hline Smith and Nephew Q Fix & 13 & 0 & \\
\hline Smith and Nephew Bioraptor & 1 & 0 & \\
\hline Smith and Nephew Healicoil & 1 & 0 & \\
\hline
\end{tabular}

Table 3 Tonnis osteoarthritis classification and Outerbridge acetabular and femoral chondromalacia classifications

\begin{tabular}{|l|l|l|l|l|l|l|l|}
\hline & Group & $\mathbf{0}$ & $\mathbf{1}$ & $\mathbf{2}$ & $\mathbf{3}$ & $\mathbf{4}$ & $\mathbf{p}$-Value \\
\hline \multirow{2}{*}{$\begin{array}{l}\text { Hip osteoarthritis } \\
\text { Tonnis grade }\end{array}$} & Doxycycline & 37 & 13 & 0 & 0 & 0 \\
\cline { 2 - 8 } & No doxycycline & 66 & 18 & 0 & 0 & 0 \\
\hline $\begin{array}{l}\text { Acetabular chondromalacia } \\
\text { Outerbridge grade }\end{array}$ & Doxycycline & 5 & 5 & 32 & 3 & 5 & 0.152 \\
\cline { 2 - 8 } & No doxycycline & 15 & 1 & 52 & 5 & 9 & 0.716 \\
\hline $\begin{array}{l}\text { Femoral chondromalacia } \\
\text { Outerbridge grade }\end{array}$ & Doxycycline & 41 & 5 & 2 & 2 & 0 \\
\cline { 2 - 9 } & No doxycycline & 64 & 5 & 5 & 6 & 1 \\
\hline
\end{tabular}

tetracycline, minocycline, has already been well established in its effectiveness for rheumatoid arthritis. ${ }^{21-25}$ In addition, tetracyclines' ability to inhibit microglial cell and astrocyte activation has shown the potential to decrease chronic pain in an animal model of osteoarthritis. ${ }^{11}$

Our analyses suggested doxycycline was not effective in combating postoperative pain. Overall, the groups did not differ significantly in any postoperative PROs, including the proportion of those who reported outcomes above the PASS threshold $\left(\right.$ HOOS $\left._{\text {global }}>62.5\right),{ }^{18}$ the iHOT VAS, and reoperation rate. The treatment groups did differ in preoperative MCSs, which could have served as a confounder causing the outcomes of the doxycycline group to be suppressed postoperatively. Chronic hip pain and postoperative pain scores have been shown to be significantly correlated with depression. ${ }^{26,27}$ As such, the experimental group would be expected to experience more postoperative pain and therefore report lower functional scores.

Despite the overall difference in preoperative MCSs between groups, subgroup analysis of only those with low preoperative MCSs also showed no difference in postoperative outcomes between groups. This finding suggests that the doxycycline group's insignificantly different results in the overall analysis were not due to preoperative MCS differences. Furthermore, the nondoxycycline group exhibited higher preoperative $\alpha$ angles on average and had fewer anchors placed on average. While a higher $\alpha$ angle suggests the potential for more severe FAIS, the fewer anchors placed may suggest less difficult labral tear fixation intraoperatively. While it is difficult to fully isolate doxycycline as an independent variable, our findings suggest that, regardless of preoperative symptoms, "acceptable" symptomatic 
Table 4 Overall group analyses

\begin{tabular}{|c|c|c|c|c|c|}
\hline \multirow[b]{2}{*}{ Metric } & \multicolumn{2}{|l|}{ Doxycycline } & \multicolumn{2}{|l|}{ No doxycycline } & \multirow[t]{2}{*}{$p$-Value } \\
\hline & Mean \pm SD & $N$ & Mean \pm SD & $N$ & \\
\hline Reoperation & - & 8 & - & 8 & 0.399 \\
\hline Pre-op $\alpha$ angle & $55.4 \pm 10.5$ & 48 & $60.0 \pm 13.9$ & 76 & 0.052 \\
\hline Post-op $\alpha$ angle & $44.3 \pm 7.4$ & 48 & $47.5 \pm 9.9$ & 73 & 0.065 \\
\hline Pre-op LCEA & $33.0 \pm 7.0$ & 49 & $31.9 \pm 6.8$ & 79 & 0.384 \\
\hline Post-op LCEA & $31.8 \pm 6.5$ & 49 & $31.2 \pm 6.8$ & 79 & 0.619 \\
\hline Number of anchors & $3.7 \pm 1.8$ & 42 & $3.2 \pm 1.2$ & 71 & 0.056 \\
\hline Size of labral defect & $9: 52$ to $2: 20$ & 50 & $10: 45$ to $1: 23$ & 78 & 0.130 \\
\hline Pre-op MCS & $38.2 \pm 17.0$ & 30 & $48.8 \pm 11.8$ & 58 & $<0.001$ \\
\hline Post-op iHOT & $52.3 \pm 24.5$ & 50 & $54.6 \pm 21.4$ & 81 & 0.590 \\
\hline Post-op iHOT VAS & $31.2 \pm 26.9$ & 45 & $34.6 \pm 23.5$ & 80 & 0.463 \\
\hline Post-op HOOS global & $58.7 \pm 14.6$ & 48 & $59.0 \pm 12.5$ & 84 & 0.910 \\
\hline$>\mathrm{HOOS}_{\text {global }}$ PASS threshold & - & 13 & - & 28 & 0.580 \\
\hline
\end{tabular}

Abbreviations: $\mathrm{HOOS}_{\text {global }}$, Hip Disability and Osteoarthritis Outcome Score (HOOS) global; iHOT, International Hip Outcome Tool; LCEA, lateral center-edge angle; MCS, mental component score; PASS, patient-acceptable symptom state; VAS, visual analogue scale. Bold values highlight statistical significance.

Table 5 Subgroup analyses of patients with preoperative MCS less than 42.9

\begin{tabular}{|c|c|c|c|c|c|}
\hline \multirow[b]{2}{*}{ PRO } & \multicolumn{2}{|l|}{ Doxycycline } & \multicolumn{2}{|l|}{ No doxycycline } & \multirow[t]{2}{*}{$p$-Value } \\
\hline & Mean \pm SD & $N$ & Mean \pm SD & $N$ & \\
\hline Reoperation & 8 & 41 & 7 & 42 & 0.337 \\
\hline Pre-op $\alpha$ angle & $55.3 \pm 11.0$ & 40 & $63.5 \pm 16.1$ & 41 & 0.009 \\
\hline Post-op $\alpha$ angle & $44.3 \pm 7.5$ & 40 & $48.7 \pm 12.3$ & 40 & 0.042 \\
\hline Pre-op LCEA & $33.4 \pm 7.4$ & 41 & $31.7 \pm 8.0$ & 42 & 0.318 \\
\hline Post-op LCEA & $32.0 \pm 6.8$ & 41 & $31.6 \pm 7.5$ & 42 & 0.800 \\
\hline Number of anchors & $3.8 \pm 1.8$ & 36 & $2.9 \pm 1.3$ & 36 & 0.018 \\
\hline Size of labral defect & $10: 00$ to $2: 20$ & 50 & $10: 45$ to $1: 13$ & 40 & 0.401 \\
\hline Post-op iHOT & $53.6 \pm 23.1$ & 22 & $45.5 \pm 20.3$ & 19 & 0.250 \\
\hline Post-op iHOT VAS & $30.7 \pm 27.2$ & 38 & $36.8 \pm 26.5$ & 41 & 0.316 \\
\hline Post-op $\mathrm{HOOS}_{\text {global }}$ & $57.3 \pm 7.4$ & 21 & $51.6 \pm 13.3$ & 19 & 0.120 \\
\hline$>$ HOOS $_{\text {global }}$ PASS threshold & - & 4 & - & 4 & $>0.999$ \\
\hline
\end{tabular}

Abbreviations: HOOSglobal, Hip Disability and Osteoarthritis Outcome Score (HOOS) global; iHOT, International Hip Outcome Tool; LCEA, lateral center-edge angle; MCS, mental component score; PASS, patient-acceptable symptom state; PRO, patient-reported outcome; VAS, visual analogue scale. Bold values highlight statistical significance.

improvement was achieved in roughly the same proportions in each group.

In addition, the subgroup analysis further subverts the potential for doxycycline to be of added benefit to those patients with low MCS who would theoretically be most at risk for pain-related complications. Its added benefit was thought to be through a dual mechanism of inhibiting MMPs and potentially acting as an antidepressant via a reduction in neuroinflammation. ${ }^{28-30}$ Such a hypothesis has been supported by a placebo-controlled randomized trial with minocycline as the treatment drug. ${ }^{31}$ In addition, the chronic central nervous system inflammation now implicated in certain depression etiologies is also causative of chronic osteoarthritis pain in animal models, ${ }^{11}$ which minocycline has similarly been found to reduce. ${ }^{32}$ Our findings suggest that while tetracyclines may have future clinical utility as antineuroinflammatory drugs, doxycycline in the post-hip arthroscopy setting did not provide a secondary benefit to patients with low MCSs in the early postoperative setting.

Our study contains common limitations of retrospective designs. The PRO instruments used in our outcomes registry changed over time and, as such, many patients did not have preoperative scores available. To maintain adequate power, only postoperative outcome comparisons were included in the analysis, but we are then limited in our ability to assess pre- to postoperative changes. This was compensated for by 
using the $\mathrm{HOOS}_{\text {global }}$ PASS criteria to assess for the proportion of patients who achieved acceptable postoperative outcomes in each group. Even with this adjustment, the sample size collected allowed for determination of a minimum of over $20 \%$ difference in those achieving PASS threshold. There may exist a true difference between the groups of less than $20 \%$.

In addition, the retrospective nature of the study would potentially introduce significant selection bias, which allowed the potential for the doxycycline and no-doxycycline groups to differ. The risk of this bias was minimized by comparing the group demographics and using subgroup analysis to account for the measure in which the two groups did significantly differ. Further, the groups were not shown to differ significantly in capsular management, anchor types, or distribution of osteochondroplasty. Additionally, no patients before November 2018 received doxycycline, while all patients after November 2018 received doxycycline. Although this was a retrospective study, the doxycycline administration was in practice similar to a trial using blocked randomization. This design does lead to potential surgical skill differences, however, as hip arthroscopy complications have been shown to decrease with increased surgeon experience. ${ }^{33}$ The learning curve as described by Mehta et al suggests that the surgeon included in the present study may have advanced into a higher stratum during the course of the 134 hip arthroscopies performed, ${ }^{33}$ to improved outcomes in the more recent doxycycline group.

While these limitations may not allow for the potential benefits of postoperative doxycycline to be fully captured, our results did not demonstrate an early postoperative benefit with the use of doxycycline. Further analysis and long-term follow-up studies are indicated to evaluate for long-term benefits of doxycycline in potentiating the neuroinflammatory response associated with hip arthroscopy. Furthermore, prospective studies should analyze opioid use postoperatively with doxycycline treatment. The present study was not able to analyze opioid use due to the retrospective nature of the study, but a design prospectively including pill counts along with PROs for pain may illuminate benefit of tetracyclines, decreasing need for opioids.

\section{Conclusion}

Mitigating postoperative pain is of importance after arthroscopic FAIS procedures. Our study aimed to see if postoperatively administered doxycycline attenuated pain, resulting in better postoperative outcomes. The PRO scores did not significantly differ between those who did and did not receive a 7-day postoperative course of doxycycline. There was also no difference in the proportion of patients who achieved the postoperative $\mathrm{HOOS}_{\text {global }}$ PASS threshold. Our study suggests that doxycycline does not decrease postoperative pain in hip arthroscopy patients.

Data Availability

IRB protocol\# 47170, approved August 19, 2020.
Conflict of Interest

None declared.

\section{References}

1 Pun S, Kumar D, Lane NE. Femoroacetabular impingement. Arthritis Rheumatol 2015;67(01):17-27

2 Matsuda DK, Carlisle JC, Arthurs SC, Wierks CH, Philippon MJ. Comparative systematic review of the open dislocation, miniopen, and arthroscopic surgeries for femoroacetabular impingement. Arthroscopy 2011;27(02):252-269

3 Philippon MJ, Schenker ML, Briggs KK, Kuppersmith DA, Maxwell RB, Stubbs AJ. Revision hip arthroscopy. Am J Sports Med 2007;35 (11):1918-1921

4 Minkara AA, Westermann RW, Rosneck J, Lynch TS. Systematic review and meta-analysis of outcomes after hip arthroscopy in femoroacetabular impingement. Am J Sports Med 2019;47(02): 488-500

5 Arung W, Meurisse M, Detry O. Pathophysiology and prevention of postoperative peritoneal adhesions. World J Gastroenterol 2011;17(41):4545-4553

6 Shin JJ, McCrum CL, Mauro CS, Vyas D. Pain management after hip arthroscopy: systematic review of randomized controlled trials and cohort studies. Am J Sports Med 2018;46(13):3288-3298

7 Usher KM, Zhu S, Mavropalias G, Carrino JA, Zhao J, Xu J. Pathological mechanisms and therapeutic outlooks for arthrofibrosis. Bone Res 2019;7:9

8 Brandt KD, Mazzuca SA, Katz BP, et al. Effects of doxycycline on progression of osteoarthritis: results of a randomized, placebocontrolled, double-blind trial. Arthritis Rheum 2005;52(07): 2015-2025

9 Farhad SZ, Aminzadeh A, Mafi M, Barekatain M, Naghney M, Ghafari MR. The effect of adjunctive low-dose doxycycline and licorice therapy on gingival crevicular fluid matrix metalloproteinase-8 levels in chronic periodontitis. Dent Res J (Isfahan) 2013;10(05):624-629

10 Castro MM, Tanus-Santos JE, Gerlach RF. Matrix metalloproteinases: targets for doxycycline to prevent the vascular alterations of hypertension. Pharmacol Res 2011;64(06):567-572

11 Sagar DR, Burston JJ, Hathway GJ, et al. The contribution of spinal glial cells to chronic pain behaviour in the monosodium iodoacetate model of osteoarthritic pain. Mol Pain 2011;7:88

12 Rosenblum A, Landy DC, Perrone MA, Whyte N, Kang R. The presence of a psychiatric condition is associated with undergoing hip arthroscopy for femoroacetabular impingement: a matched case-controlled study. J Arthroplasty 2019;34(03):446-449

13 Stone AV, Malloy P, Beck EC, et al. Predictors of persistent postoperative pain at minimum 2 years after arthroscopic treatment of femoroacetabular impingement. Am J Sports Med 2019; 47(03):552-559

14 Jacobs CA, Burnham JM, Jochimsen KN, Molina D IV, Hamilton DA, Duncan ST. Preoperative symptoms in femoroacetabular impingement patients are more related to mental health scores than the severity of labral tear or magnitude of bony deformity. J Arthroplasty 2017;32(12):3603-3606

15 Selim AJ, Rogers W, Fleishman JA, et al. Updated U.S. population standard for the Veterans RAND 12-item Health Survey (VR-12). Qual Life Res 2009;18(01):43-52

16 Clohisy JC, Carlisle JC, Beaulé PE, et al. A systematic approach to the plain radiographic evaluation of the young adult hip. J Bone Joint Surg Am 2008;90(Suppl 4):47-66

17 Mittal A, Bomar JD, Jeffords ME, Huang MT, Wenger DR, Upasani VV. Defining the lateral edge of the femoroacetabular articulation: correlation analysis between radiographs and computed tomography. J Child Orthop 2016;10(05):365-370

18 Jacobs CA, Peabody MR, Duncan ST, et al; ANCHOR Group. Development of the $\mathrm{HOOS}_{\text {global }}$ to assess patient-reported 
outcomes in patients undergoing hip preservation procedures. Am J Sports Med 2018;46(04):940-946

19 R: A Language and Environment for Statistical Computing. R Foundation for Statistical Computing; 2010. Available at: http:// www.R-project.org

20 Bastos LF, de Oliveira AC, Watkins LR, Moraes MF, Coelho MM. Tetracyclines and pain. Naunyn Schmiedebergs Arch Pharmacol 2012;385(03):225-241

21 Tilley BC, Alarcón GS, Heyse SP, et al; MIRA Trial Group. Minocycline in rheumatoid arthritis. A 48-week, double-blind, placebocontrolled trial. Ann Intern Med 1995;122(02):81-89

22 O'Dell JR, Blakely KW, Mallek JA, et al. Treatment of early seropositive rheumatoid arthritis: a two-year, double-blind comparison of minocycline and hydroxychloroquine. Arthritis Rheum 2001;44(10):2235-2241

23 O'Dell JR, Haire CE, Palmer W, et al. Treatment of early rheumatoid arthritis with minocycline or placebo: results of a randomized, double-blind, placebo-controlled trial. Arthritis Rheum 1997;40 (05):842-848

24 O'Dell JR, Paulsen G, Haire CE, et al. Treatment of early seropositive rheumatoid arthritis with minocycline: four-year followup of a double-blind, placebo-controlled trial. Arthritis Rheum 1999;42 (08):1691-1695

25 Stone M, Fortin PR, Pacheco-Tena C, Inman RD. Should tetracycline treatment be used more extensively for rheumatoid arthri- tis? Metaanalysis demonstrates clinical benefit with reduction in disease activity. J Rheumatol 2003;30(10):2112-2122

26 Schwarze M, Häuser W, Schmutzer G, Brähler E, Beckmann NA Schiltenwolf M. Obesity, depression and hip pain. Musculoskelet Care 2019;17(01):126-132

27 Ghoneim MM, O'Hara MW. Depression and postoperative complications: an overview. BMC Surg 2016;16:5

28 Berk M, Williams LJ, Jacka FN, et al. So depression is an inflammatory disease, but where does the inflammation come from? BMC Med 2013;11:200

29 Dean OM, Data-Franco J, Giorlando F, Berk M. Minocycline: therapeutic potential in psychiatry. CNS Drugs 2012;26(05):391-401

30 Holmes SE, Hinz R, Conen S, et al. Elevated translocator protein in anterior cingulate in major depression and a role for inflammation in suicidal thinking: a positron emission tomography study. Biol Psychiatry 2018;83(01):61-69

31 Cai DB, Zheng W, Zhang QE, et al. Minocycline for depressive symptoms: a meta-analysis of randomized, double-blinded, placebo-controlled trials. Psychiatr Q 2020;91(02):451-461

32 Shultz RB, Zhong Y. Minocycline targets multiple secondary injury mechanisms in traumatic spinal cord injury. Neural Regen Res 2017;12(05):702-713

33 Mehta N, Chamberlin P, Marx RG, et al. Defining the learning curve for hip arthroscopy: a threshold analysis of the volume-outcomes relationship. Am J Sports Med 2018;46(06):1284-1293 